\title{
Chronic Khat (Catha edulis) Ingestion as a Possible Triggering Agent in the Development of Autoimmune Hepatitis
}

\author{
John Samies ${ }^{\mathrm{a}, \mathrm{d}}$, Sanjeev Slehria ${ }^{\mathrm{b}}$, Xiao-Lan Chen ${ }^{\mathrm{c}}$, Satyanarayana Vaidya ${ }^{\mathrm{c}}$, \\ Nafisa Saleem ${ }^{c}$
}

\begin{abstract}
We describe the case of a 34-year-old immigrant male from Yemen who presented with liver dysfunction secondary to chronic ingestion of khat, a commonly abused plant found in East Africa and the Arabian Peninsula. The clinical features of this case, in combination with the diagnostic labs, were consistent with a presentation of a khatinduced hepatotoxicity in the form of an advanced autoimmune process. Khat has already been identified in recent literature and case reports to be a hepatotoxic environmental agent, and new literature has now recognized an increased association between khat consumption and the development of autoimmune hepatitis, although no specific mechanism for triggering an autoimmune response has been elucidated. Our patient exhibited biochemical, immunological, and histopathological features of autoimmune hepatitis, which we believe may have precipitated from the patient's chronic khat drug consumption.
\end{abstract}

Keywords: Khat; Khat-induced; Hepatotoxic; Autoimmune hepatitis; Yemen

\section{Introduction}

Khat chewing is a popular social habit among the Yemeni, Somali, and East African people [1]. The popularity for chewing khat is primarily due to the psycho-stimulant effects that the use of khat produces. Until recently, the use of khat has been largely confined to East Africa and the Arabian Peninsula, and little has been known about khat-induced hepatitis or khat-induced chronic liver disease. Due to immigration, this social habit has been steadily gaining popularity in Western

\footnotetext{
Manuscript accepted for publication August 10, 2016

a Jerry Wallace Campbell University School of Osteopathic Medicine, Lillington, NC, USA

${ }^{\mathrm{b}}$ Cape Fear Center for Digestive Diseases, Fayetteville, NC, USA

${ }^{\mathrm{c}}$ Cape Fear Valley Medical Center, Fayetteville, NC, USA

${ }^{\mathrm{d} C o r r e s p o n d i n g ~ A u t h o r: ~ J o h n ~ S a m i e s, ~ C a m p b e l l ~ U n i v e r s i t y ~ S c h o o l ~ o f ~ O s t e o-~}$ pathic Medicine, Leon Levine Hall of Medical Sciences, 4350 US-421, Li1lington, NC 27546, USA. Email: jhsamies0225@email.campbell.edu
}

doi: http://dx.doi.org/10.14740/jmc2598w countries [2]. To our knowledge, this is the first documented case of khat-induced hepatotoxicity in the US, and one of only a small number of documented cases involving the formation of autoimmune hepatitis (AIH).

\section{Case Report}

A 34-year-old immigrant male from Yemen with a medical history significant for kidney stones, ureteral stones, and jaundice in the past presented to the emergency department with a complaint of yellow discoloration of the eyes, dark urine, and bothersome pruritus. The patient was of a usual state of health until about 3 weeks prior when he first noticed the progressive jaundice. The patient had additional complaints of intermittent right upper quadrant abdominal pain and intermittent fever, although no temperature was taken at home. Further discussion with the patient revealed a similar episode of jaundice 3 years prior where he was initially evaluated in the emergency department, but subsequently left the hospital against medical advice without a clear diagnosis. The previous episode of jaundice was said to have resolved spontaneously without any additional medical intervention.

At initial presentation, the patient denied alcohol and drug abuse, use of over-the-counter medications, or use of any herbal supplements. His physical examination was significant only for scleral icterus, hepatomegaly, and mild-to-moderate splenomegaly.

Laboratory investigations for the patient suggested a pattern of acute hepatitis with the following results: total bilirubin $15.8 \mathrm{mg} / \mathrm{dL}$ (reference range (RR): $0.2-1.0 \mathrm{mg} / \mathrm{dL}$ ), direct bilirubin $13.01 \mathrm{mg} / \mathrm{dL}$ (RR: $<0.2 \mathrm{mg} / \mathrm{dL}$ ), aspartate aminotransferase $689 \mathrm{U} / \mathrm{L}$ (RR: 15 - $37 \mathrm{U} / \mathrm{L}$ ), alanine aminotransferase $935 \mathrm{U} / \mathrm{L}$ (RR: 12 - $78 \mathrm{U} / \mathrm{L}$ ), and alkaline phosphatase 209 U/L (RR: 45 - 117 U/L). Results of the complete blood count, serum electrolyte levels, and coagulation studies were normal. Viral serology labs for hepatitis A, B, C, and E were all negative, and human immunodeficiency virus infection was excluded as well. Laboratory workup also did not show any evidence of hemochromatosis, Wilson disease, or alpha-1 antitrypsin deficiency. Mitochondrial antibody and anti-nuclear antibody direct testing were negative. However, anti-smooth muscle antibody and IgG antibody counts were shown to have strong positivity, at levels of 53 units (RR: $<19$ units) and 

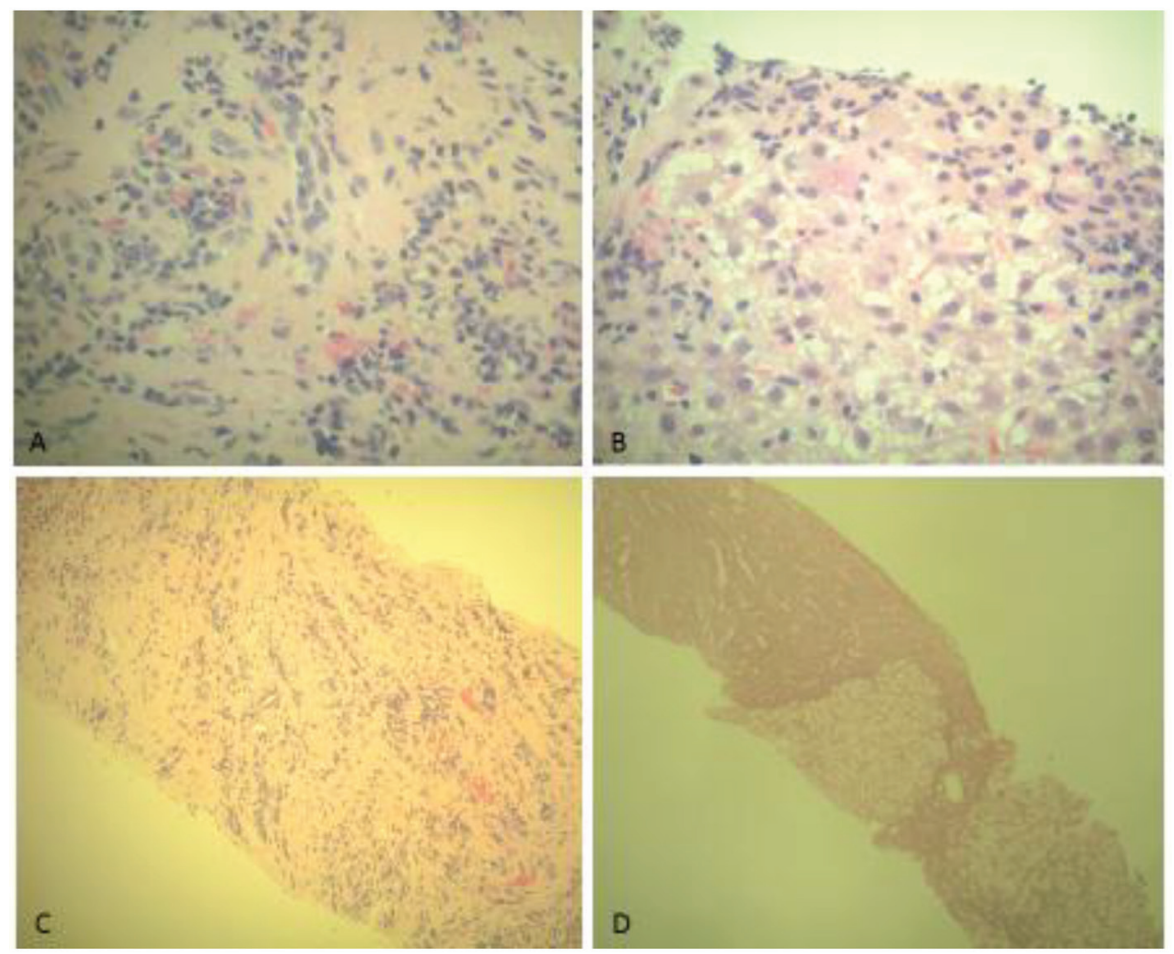

Figure 1. (A, B) H\&E stain of liver biopsy demonstrating interface hepatitis and diffuse infiltration by mixed inflammatory cells including neutrophils, lymphocytes, plasma cells, and eosinophils. (C, D) H\&E stain of liver biopsy demonstrating portal tract infiltration by mixed inflammatory cells and portal tract expansion by fibrosis in focal areas. (D) Regenerative nodularity of the liver biopsy, indicating probable liver cirrhosis.

$2,634 \mathrm{mg} / \mathrm{dL}(\mathrm{RR}:<1,600 \mathrm{mg} / \mathrm{dL})$, respectively, indicating a high probability of an underlying AIH.

Initial imaging via ultrasonography and computed tomography showed hepatomegaly, splenomegaly, and possible cirrhotic liver changes. A patent portal vein was visualized and there was no obvious evidence of biliary ductal dilation. A thorough assessment of the biliary tree was made via magnetic resonance cholangiopancreatography (MRCP), which confirmed the absence of an obstructive process. A percutaneous core needle liver biopsy was performed on the fourth day of hospital admission. Liver biopsy concluded a chronic hepatitis with grade IV inflammation. Piecemeal and lobular necrosis was visualized. Stage III-IV focal fibrosis and sporadic bridging fibrosis was present. There was evidence of hepatic steatosis with focal ballooning changes. The liver portal tracts were diffusely infiltrated by mixed inflammatory cells including neutrophils, plasma cells, lymphocytes, and eosinophils (Fig. 1). Taking the patient's clinical presentation, negative viral panel, and positive autoimmune markers into account, the processed liver biopsy samples suggested features of AIH with a differential diagnosis of drug-induced hepatitis.

With supportive management, the patient experienced moderate liver function improvement. At the time of hospital discharge, the patient's total bilirubin had decreased to $7.1 \mathrm{mg} /$ $\mathrm{dL}$, and the patient's aspartate aminotransferase and alanine aminotransferase were 430 and $576 \mathrm{U} / \mathrm{L}$, respectively. At this time, the patient's brother disclosed information pertaining to the patient's long-term intermittent use of khat for recreational purposes. This information was then confirmed with the patient. The patient was instructed to immediately abstain from the use of khat and was started on $40 \mathrm{mg}$ prednisone daily. At 1-week post-discharge follow-up, the patient admitted overall improvement of health status and labs continued to show liver function improvement.

\section{Discussion}

Khat (Catha edulis) is a slow-growing flowering shrub native to portions of East Africa and the Arabian Peninsula [1, 2]. When chewed, khat leaves release an amphetamine-like substance which produces a pleasurable euphoric effect. As a popular cultural custom, khat is estimated to be used daily by over 20 million people in Yemen, Somalia, and other surrounding countries [2]. This number is steadily increasing due to the introduction of khat chewing to Western countries through extensive immigration. Populations of Yemeni and Somali immigrants living in the UK and the US have contributed to the spread this popular social habit, making khat use a worldwide issue $[2,3]$.

The alkaloid compounds primarily responsible for the stimulant properties of khat include cathinone, and to a lesser degree, cathine, and norephedrine. The main alkaloid, cathinone, has been shown to have considerable structural similarity with amphetamine $[3,4]$, leading to a similar central nervous system response when ingested. It is also relatively 
unstable, and will spontaneously break down into cathine and norephedrine over a short period of time after the khat leaves are harvested. Therefore, only fresh khat leaves seem to hold the most potency $[2,4]$.

Cathinone acts on the central nervous system to release large stores of dopamine and other catecholamines. When chewed, khat typically evokes an initial feeling of excitability, increased alertness, and euphoria $[3,5]$. This stimulation is typically followed by unpleasant feelings of lethargy, somnolence, and sadness. For chronic users and heavy users, severe depression and psychosis may occur $[3,6]$. As with amphetamine use, khat users are at increased risk for major cardiovascular events. Typical adverse events from khat consumption include anxiety, anorexia, hypertension, hyperthermia, and mydriasis, as well as recurring gastrointestinal episodes of esophagitis, gastritis, ulcers, and constipation [3, 5-7].

With khat use becoming more prevalent, there has been an increased awareness and growing concern about the potential for khat-induced hepatotoxic injury [1, 6-13]. Animal studies published in 2002 have evaluated the toxicological effects of long-term khat ingestion and have shown significant hepatotoxic injury from khat exposure in rabbits [7, 8]. The hepatotoxic features from these studies revealed marked increases in the plasma concentrations of alkaline phosphatase and liver transaminases, along with steady increases in direct and total bilirubin levels. Additionally, histopathological analysis from these studies revealed mixed inflammatory infiltrate and portoportal fibrosis $[7,8]$. Human studies have corroborated this evidence through an increasing number of case studies published from Europe and Australia on khat-induced hepatotoxicity in Yemeni and Somali immigrants [9-13].

In addition to hepatotoxic effects, khat has now gained recognition as a potential triggering agent in the development of a severe form of AIH [2, 14, 15]. AIH develops from a breakdown of the body's immunological tolerance to antigens found on hepatocytes. This breakdown of tolerance is often compounded by a loss of appropriate immune system regulation, leaving the immune system to mount extensive immune responses against the liver [15-17]. Recent studies have suggested that chronic exposure to khat is triggering AIH in individuals via an unknown mechanism [15]. Although no specific cause to the development of AIH in chronic khat users has been elucidated, a number of studies have explored the potential connection of khat inducing AIH in genetically susceptible individuals $[2,15]$, as well as the potential ability for khat to trigger drug-induced immune responses through auto-antigen mimicry [15].

In a case study of three Yemeni patients with a social history of chronic daily khat use, all three patients had a positive immunological profile consistent with type one AIH. These patients shared similarities in their clinical presentation and diagnostic workup, and all three had a favorable response to steroid treatments [14]. In a second study exploring the potential role of khat in triggering $\mathrm{AIH}$, six patients were identified from the Hepatology database at Sheffield Hospital system in the UK as having acute hepatitis with a background of khat use. These patients were evaluated for the probability of having AIH and a strong argument was made that these patients developed a suspected atypical presentation of $\mathrm{AIH}$ through khat-induced hepatotoxic effects and possible genetic susceptibility [2]. This evidence was supported by an earlier case study from the UK that identified seven Somali patients from 1996 to 2006 who presented with acute hepatitis and a background significant for chronic khat use. Two out of the seven patients in this study were found to have met the criteria for probable AIH [10]. Atypical presentations of AIH in Somali men have also been documented in an additional clinical study [18], and although no specific mention of khat use was reported, it is believed that there may be a correlation between the increased presentations of AIH seen in Somali men and the prevalent use and underreporting of khat consumption in this region $[1,2,10]$.

$\mathrm{AIH}$ is a chronic inflammatory condition of unknown etiology and is typically reserved as a diagnosis of exclusion [1618]. It is characterized by elevated liver transaminase levels, a positive autoantibody screen, elevated immunoglobulin G levels, and interface hepatitis on histology [16-18]. The clinical course of AIH may be characterized by a waxing and waning of autoimmune activity, making clinical manifestations among patients decidedly variable [16]. AIH can be triggered through exposure to environmental agents, drugs, and cross-reactivity between viral particles and liver antigens. It is unclear whether drugs, herbs, and other environmental agents expose or induce $\mathrm{AIH}$, or if they cause a drug-induced hepatitis with autoimmune features [16]. Our patient has demonstrated characteristic features of AIH with a background significant for khat consumption. It is hypothesized that chronic chewing of khat induces a form of AIH in genetically susceptible individuals, and current literature helps to support this association $[2,14]$. Our hypothesis that khat induces AIH is further strengthened by the lack of AIH cases within Somali women, as fewer women seem to engage in khat chewing [10]. From the current literature, the prognosis of khat-induced liver injury is unclear. Favorable responses to abstinence from khat and corticosteroid therapy have been documented $[2,14]$, although progression to cirrhosis or fulminant liver failure from drug-induced hepatotoxicity is possible [13].

The implication that khat consumption causes $\mathrm{AIH}$ in genetically susceptible individuals should only be used as a tentative interpretation based on current literature and our patient's clinical and diagnostic presentation. More research is needed to better elucidate the role of khat in the pathogenesis of AIH. This study highlights a need for better cultural awareness in the US when dealing with immigrants from East Africa and the Arabian Peninsula who present with unique forms of hepatitis, cirrhosis, or fulminant liver failure.

\section{Disclosures}

There are no conflicts of interest or financial support involved in this case study.

\section{Author Note}

Sanjeev Slehria, Xiao-Lan Chen, Satyanarayana Vaidya, and Nafisa Saleem are contracted through Cape Fear Valley Medi- 
cal Center, 1638 Owen Drive, Fayetteville, NC, USA.

\section{References}

1. Forbes MP, Raj AS, Martin J, Lampe G, Powell EE. Khatassociated hepatitis. Med J Aust. 2013;199(7):498-499.

2. Riyaz S, Imran M, Gleeson D, Karajeh MA. Khat (Catha Edulis) as a possible cause of autoimmune hepatitis. World J Hepatol. 2014;6(3):150-154.

3. Cox G, Rampes H. Adverse effects of khat: a review. Advances in Psychiatric Treatment. 2003;9(6):456-463.

4. Kalix P. Cathinone, a natural amphetamine. Pharmacol Toxicol. 1992;70(2):77-86.

5. Brenneisen R, Fisch HU, Koelbing U, Geisshusler S, Kalix P. Amphetamine-like effects in humans of the khat alkaloid cathinone. Br J Clin Pharmacol. 1990;30(6):825828 .

6. Roelandt P, George C, d'Heygere F, Aerts R, Monbaliu D, Laleman W, Cassiman D, et al. Acute liver failure secondary to khat (Catha edulis)-induced necrotic hepatitis requiring liver transplantation: case report. Transplant Proc. 2011;43(9):3493-3495.

7. Al-Habori M, Al-Aghbari A, Al-Mamary M, Baker M. Toxicological evaluation of Catha edulis leaves: a long term feeding experiment in animals. J Ethnopharmacol. 2002;83(3):209-217.

8. Al-Mamary M, Al-Habori M, Al-Aghbari AM, Baker MM. Investigation into the toxicological effects of Catha edulis leaves: a short term study in animals. Phytother
Res. 2002;16(2):127-132.

9. Brostoff JM, Plymen C, Birns J. Khat - a novel cause of drug-induced hepatitis. Eur J Intern Med. 2006;17(5):383.

10. Peevers CG, Moorghen M, Collins PL, Gordon FH, McCune CA. Liver disease and cirrhosis because of Khat chewing in UK Somali men: a case series. Liver Int. 2010;30(8):1242-1243.

11. Patanwala IM, Burt AD, Bassendine MF, Hudson M. Khat associated end stage chronic liver disease: a case report. Journal of Medical Cases. 2011;2(3):104-106.

12. Chapman MH, Kajihara M, Borges G, O'Beirne J, Patch D, Dhillon AP, Crozier A, et al. Severe, acute liver injury and khat leaves. N Engl J Med. 2010;362(17):1642-1644.

13. Stuyt RJ, Willems SM, Wagtmans MJ, van Hoek B. Chewing khat and chronic liver disease. Liver Int. 2011;31(3):434-436.

14. Fallatah HI, Akbar HO. Qat chewing and autoimmune hepatitis - true association or coincidence. Middle East Journal of Family Medicine. 2010;8(3):6-9.

15. Fallatah HI, Akbar HO. Autoimmune hepatitis as a unique form of an autoimmune liver disease: immunological aspects and clinical overview. Autoimmune Diseases. 2012;2012:17.

16. Krawitt EL. Autoimmune hepatitis. N Engl J Med. 2006;354(1):54-66.

17. Czaja AJ, Freese DK. Diagnosis and treatment of autoimmune hepatitis. Hepatology. 2002;36(2):479-497.

18. D'Souza R, Sinnott P, Glynn MJ, Sabin CA, Foster GR. An unusual form of autoimmune hepatitis in young Somalian men. Liver Int. 2005;25(2):325-330. 